Article

\title{
Nanoscale Sensing Using Point Defects in Single-Crystal Diamond: Recent Progress on Nitrogen Vacancy Center-Based Sensors
}

\author{
Ettore Bernardi, Richard Nelz, Selda Sonusen and Elke Neu * \\ Faculty for Natural Sciences and Technology, Physics Department, Saarland University, 66123 Saarbrücken, \\ Germany; ettore.bernardi@physik.uni-saarland.de (E.B.); richard.nelz@uni-saarland.de (R.N.); \\ selda.sonusen@physik.uni-saarland.de (S.S.) \\ * Correspondence: elkeneu@physik.uni-saarland.de; Tel.: +49-681-302-2739
}

Academic Editor: Yuri Palyanov

Received: 8 April 2017; Accepted: 24 April 2017; Published: 28 April 2017

\begin{abstract}
Individual, luminescent point defects in solids, so-called color centers, are atomic-sized quantum systems enabling sensing and imaging with nanoscale spatial resolution. In this overview, we introduce nanoscale sensing based on individual nitrogen vacancy (NV) centers in diamond. We discuss two central challenges of the field: first, the creation of highly-coherent, shallow NV centers less than $10 \mathrm{~nm}$ below the surface of a single-crystal diamond; second, the fabrication of tip-like photonic nanostructures that enable efficient fluorescence collection and can be used for scanning probe imaging based on color centers with nanoscale resolution.
\end{abstract}

Keywords: diamond; color center; magnetic sensing; scanning probes; nanostructures

\section{Introduction}

Nanotechnology has led to many significant technological and scientific advances in recent years. For instance, two-dimensional or nanoscale materials such as carbon nanotubes or graphene are investigated for next generation electronics and photonics [1]. Simultaneously, functionalized nanoparticles, e.g., for drug delivery, are promising to enhance various therapies [2]. Moreover, electronic systems like transistors are being miniaturized and controlled down to the single electron level [3].

Simultaneously to developing nanotechnology, a need for sensing techniques that work on the nanoscale has been arising to investigate nanoscale materials and to foster their further development. Quantities of interest are magnetic fields, often created as a result of electrical currents [4], electric fields [5], temperatures [6], pressure or crystal strain [7], as well as the presence of individual fluorescent markers, e.g., molecules [8]. For sensing with nanoscale spatial resolution, in general, the sensor needs to fulfill several demanding prerequisites:

- The sensor's size or active area has to be small compared to the structure under investigation. If this is not the case, spatial averaging over the detector area may mask information from the sample's nanostructure. Consequently, sensors approaching atomic dimensions $(<1 \mathrm{~nm})$ are desirable for nanoscale sensing.

- The sensor's geometry must allow for close proximity in-between the investigated object and the sensor. In most cases, controlled proximity to the sample is ensured by manufacturing the sensor in a tip-like geometry and approaching it to the sample via a scanning probe mechanism. This mechanism often keeps the force between the sample and the tip constant (for pioneering work see, e.g., [9-11]). Alternatively, the sensor can consist of a nanoparticle that is, e.g., inserted into a cell for sensing [6]. 
- The sensor needs to provide sufficient sensitivity to capture the weak signals that arise from nanoscopic or atomic objects. To illustrate this demanding point, the magnetic field of a single electron spin even at a distance of $50 \mathrm{~nm}$ amounts to only $\approx 9 \mathrm{nT}$ [12]. The field of magnetic dipoles decays with the distance $r$ from the dipole like $r^{-3}$ [13]. For the near-field energy transfer between two point-like dipoles, which is a valuable imaging resource, as well, even a $r^{-6}$ decay has to be considered [14]. Thus, bringing the sensor and the sample in close proximity is not only mandatory for high resolution, but also enables detecting weak signals from nanoscale objects.

Using individual, optically-active point defects in solids as sensors allows simultaneously fulfilling the prerequisites listed above. Moreover, such defects are versatile sensors for several quantities, including magnetic and electric fields and temperature.

Point defects alter the host crystal's periodic lattice only in one or a few neighboring lattice sites. Mostly, impurity atoms enter the crystal lattice and can form complexes with vacancies. Electrons, or more precisely the electronic wave-functions, localize at the defect within a few lattice constants and thus on atomic scales; typically within less than $1 \mathrm{~nm}$ [15]. This manifests also in the existence of such defects $\approx 1 \mathrm{~nm}$ below crystal surfaces [16]. At such distances, crystal surfaces strongly influence the defects and may cause instability [17]. Leading contenders for sensing are optically-active point defects in the wide-bandgap semiconductors diamond [13,18,19] and silicon carbide [20].

This review focuses on sensors based on diamond and especially one of the most prominent point defects in diamond, the nitrogen vacancy (NV) color center. This defect consists of a nitrogen atom replacing a carbon atom and a neighboring lattice vacancy [21]. Figure 1 summarizes its basic properties. NV centers, in general, provide long-term photo-stable fluorescence. Their bright emission with high luminescence efficiency [22] lies in the red and near-infrared spectral range and spans about $100 \mathrm{~nm}$. NV color centers form emitting electric dipoles [23]. Their emission is bright enough to allow for a straightforward detection of individual, isolated centers in a confocal fluorescence microscope. Thus, NV centers have been investigated as solid-state sources of single photons [24]. Single photons in turn are a valuable resource for nanoscale sensing, e.g., in scanning near-field optical microscopy as a nanoscopic light source [25]. Alternatively, the dipole of the NV center can interact with other dipoles and transfer energy via optical near fields (Förster resonance energy transfer (FRET) [8,26]). Via this process, NV centers can reveal the presence of other dipoles. 


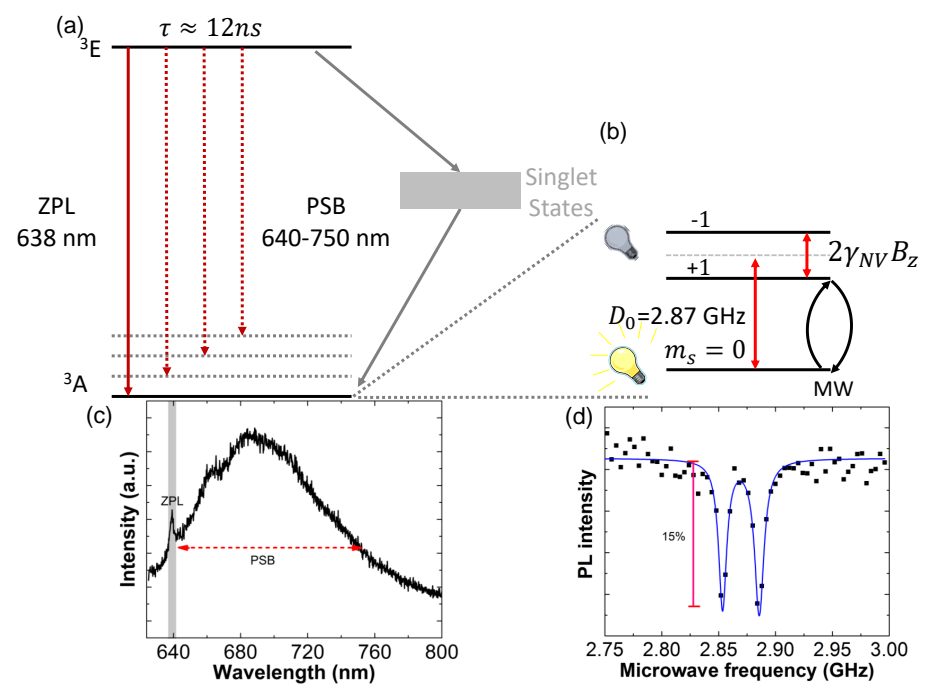

Figure 1. Illustration of the basic properties of nitrogen vacancy (NV) centers in diamond. (a) Strongly-radiative, electric dipole transitions between the NV excited $\left({ }^{3} E\right)$ and ground $\left({ }^{3} A\right)$ states create a photo-luminescence in the red and near-infrared spectral region shown in (c). For a detailed investigation of the NV level structure and dynamics, see, e.g., [27]. The excited state lifetime is $\tau \approx 12-13 \mathrm{~ns}$ in bulk diamond [28]. The purely electronic transition at $638 \mathrm{~nm}$ (zero-phonon-line (ZPL)) is marked in the spectrum in (c) and features a width of about $1 \mathrm{~nm}$ at room temperature. Transitions to vibrationally-excited states create additional, broad phonon sidebands (PSB) as indicated in the schematics and in the spectrum. (b) Detailed description of the ground state spin levels. The triplet state has three spin sub-levels: $\mathrm{m}_{s}=0$ and $\mathrm{m}_{s}= \pm 1$. If no external field is applied, the levels with the projection spin quantum number 0 and \pm 1 are split by $2.87 \mathrm{GHz}$ due to spin-spin interactions. In the presence of a magnetic field, the Zeeman effect splits the +1 and -1 states by $2 \gamma_{N V} B_{z}$ as schematically shown in (b) and discernible from the measurement in (d). Note that only the magnetic field $\mathrm{B}_{z}$ projected onto the NV's high symmetry axis (connecting line between vacancy and nitrogen, $<111>$ direction) leads to a splitting [13]. If the NV center spin is in one of the \pm 1 states, the probability is enhanced that the center undergoes an inter-system relaxation to the singlet levels (see (a)). As these levels have a lifetime that is more than one order of magnitude longer than for the triplet levels [29], the NV's photo-luminescence is reduced in the \pm 1 states enabling the optical read-out of the NV spin state (optically-detected magnetic resonance (ODMR)). For sensing applications, transitions between the 0 state and +1 or -1 state are typically driven using circularly-polarized microwaves (MW) $[23,30]$. Using green laser light $(532 \mathrm{~nm})$, the NV center is initialized to its $m_{s}=0$ state via optical pumping within roughly $1 \mu \mathrm{s}$ [31].

In addition to their optical properties, NV centers provide highly-coherent, optically-readable electronic spins (see Figure 1; first observation in [32]). The NV centers' long spin coherence times $\left(T_{2}^{*}\right.$ or $T_{2}$ ) show that coherent superposition states of the 0 and \pm 1 ground state spin levels retain their phase for a long time even at room temperature. Here, NV centers profit from the fact that the diamond lattice naturally features a low magnetic noise. Thus, it protects these superposition states from decoherence: the most abundant carbon isotope ${ }^{12} \mathrm{C}$ does not have a nuclear spin. Additionally, the concentration of paramagnetic ${ }^{13} \mathrm{C}$ isotopes $(1.1 \%)$ can be reduced by isotopically pure diamond synthesis [33]. In such an isotopically-engineered diamond, $\mathrm{T}_{2}$ can be as high as $1.8 \mathrm{~ms}$. The NV center's $T_{2}$ is a valuable sensing resource: its decrease, for example, directly reveals the presence of magnetic molecules on the diamond surface [34]. The use of spin coherence as a sensing resource opens up novel sensing schemes with potentially enhanced sensitivity compared to classical techniques. These novel approaches are typically summarized under the term of quantum sensing (for a recent review, see, e.g., [35]). However, sensing approaches that rely on the direct measurement of spin resonances (optically-detected magnetic resonance (ODMR); for an explanation, see Figure 1) and their 
shift in magnetic fields (Figure 1) also profit from highly-coherent NV centers: the NV's sensitivity $\eta$ to static magnetic fields is given by [13]:

$$
\eta \approx \frac{h}{g \mu_{B}} \frac{\Delta v}{\sqrt{I_{0} C}}
$$

where $\Delta v$ is the ODMR linewidth and $I_{0}$ is the detected photon rate from the NV center. $C$ is the fluorescence contrast in ODMR. The latter is an intrinsic property of NV centers and can hardly be modified as it is determined by the internal dynamics. $\Delta v$ is fundamentally limited by the inverse of the coherence time $\mathrm{T}_{2}^{*}$ and thus connects the sensitivity to the coherence properties.

Note that Equation (1) refers to non-resonant spin read-out, typically using green laser light to excite the NV center. This read-out scheme is feasible at room temperature and thus advantageous for sensing. At cryogenic temperature, resonantly-addressing spin-selective transitions within the ZPL enables single-shot read-out of the electronic spin state of NV centers [36].

The carbon lattice of diamond itself provides only a weak source of decoherence. However, paramagnetic impurities (e.g., nitrogen in its substitutional form) and spins on the diamond surface can significantly reduce the coherence time of NV centers [37]. Reduced coherence times render NV centers less sensitive for magnetic fields, as discernible from Equation (1). Consequently, it is vital to control this loss of coherence to use NV centers as highly-sensitive sensors for fields outside the diamond crystal.

In essence, realizing an optimal NV sensor narrows to three main aspects:

- It is mandatory to reliably create stable NV centers with a controlled density buried less than $\approx 10 \mathrm{~nm}$ below diamond surfaces (shallow NV centers). These NV centers need to retain spin coherence for optimal sensitivity.

- ODMR and optical sensing schemes demand efficient collection of fluorescence light and high photon rates from single centers. This in turn demands the incorporation of color centers into nanophotonic structures.

- To realize a sensor that probes the sample surface and to realize controlled positioning of the NV sensor requires realizing a tip-like sensor and scanning probe sensing schemes.

This review is structured according to these main aspects. In Section 2, we summarize recent progress in creating and optimizing shallow NV centers. In Section 3, we turn to the photonic nanostructures for sensing and their fabrication. In Section 4, we illustrate scanning probe-based approaches, as well as recent advances in NV-based sensing.

\section{Shallow NV Centers for Sensing}

\subsection{Creation Methods and Creation Yield}

NVs with a controlled distance to diamond surfaces have been created using two approaches, namely ion implantation and $\delta$-doping. We discuss both in the following:

- Ion implantation: This approach relies on the commercial availability of chemical vapor deposition (CVD) diamond with low nitrogen $(\mathrm{N})$ content $(<5 \mathrm{ppb}$ substitutional $\mathrm{N})$ and an almost negligible density of in situ-created, native NV centers ([38], element six, electronic grade diamond). This diamond is irradiated with $\mathrm{N}$ ions at energies typically between 4 and $8 \mathrm{keV}$. These implantation energies correspond to mean implantation depths between $\approx 7 \mathrm{~nm}$ and $\approx 12 \mathrm{~nm}$, as calculated by Monte Carlo simulations [39]. Coherence times $\mathrm{T}_{2}$ of $\mathrm{NVs}$ created deep $(>50 \mathrm{~nm})$ inside this material by $\mathrm{N}$ implantation reach $\mathrm{T}_{2} \approx 200 \mu$ s comparable to in situ-created $\mathrm{NV}$ centers [40]. Such $\mathrm{T}_{2}$ times indicate the high purity of the material, whereas in diamonds with $100 \mathrm{ppm} \mathrm{N}, \mathrm{T}_{2}$ generally reduces to about $1 \mu \mathrm{s}$ [13]. After the implantation, high temperature annealing repairs crystal damage, mobilizes vacancies via diffusion and forms $\mathrm{NV}$ complexes via $\mathrm{N}$ impurities capturing vacancies. In contrast, impurities like, for example, $\mathrm{N}$ are not expected to diffuse at these temperatures [16]. Two approaches are reported: annealing in vacuum at 
pressures below $5 \times 10^{-7}$ mbar [16,40,41], where a high vacuum is needed to avoid etching of the surface [42]. Alternatively, annealing in forming gas $\left(4 \% \mathrm{H}_{2}\right.$ in $\left.\mathrm{Ar}\right)$ is used (e.g., [43,44]). The conversion efficiency from implanted $\mathrm{N}$ to $\mathrm{NV}$ (mostly $\mathrm{NV}^{-}$) is called yield. It amounts to typically only $1 \%$ for an implantation energy of $5 \mathrm{keV}$ (depth $\approx 8 \mathrm{~nm}$ ) [41,45]. For micrometer-deep implanted NVs $(E=18 \mathrm{MeV})$, the yield increases to $45 \%$ [45]. For shallow NVs, vacancies are partially captured by the diamond surface [42]. Furthermore, higher energy implantation increases the number of vacancies produced [45]. Very recent studies, however, indicate that increasing the number of vacancies by co-implanting, e.g., carbon, does not increase the NV yield for shallow implantation [46].

- $\quad$ The $\delta$-doping method for creating shallow NVs consists of the following steps; see Figure 2:

- An N-doped, several nm-thick layer, the $\delta$-doped layer $[47,48]$, is created in situ by the controlled introduction of $\mathrm{N}_{2}$ gas during slow, plasma-enhanced CVD growth of single-crystal diamond (growth rate $\approx 0.1 \mathrm{~nm} / \mathrm{min}$; Figure $2 \mathrm{a}$ ). Changing the $\mathrm{N}_{2}$ flow tunes the resulting $\mathrm{N}$ densities. In order to decrease the magnetic noise, diamond growth can be performed using isotopically-purified ${ }^{12} \mathrm{CH}_{4}$ as the carbon source $[47,49,50]$. Very recently, overgrowth of a nitrogen-terminated diamond surface has been employed for $\delta$-doping [51].

- Vacancies are created ex situ by implanting helium ions [52,53], carbon ions [49] or irradiating with electrons $[47,50]$. A subsequent annealing at high temperature causes vacancy diffusion and creates NV centers (Figure 2c). Varying the thickness of an undoped diamond layer, overgrown before the implantation step, allows controlling the depth of the NV layer (Figure 2b). The vacancy profile depends on the species used: for electron irradiation, it is flat and extends throughout the grown film and substrate [49]. In contrast, implanted carbon ions are localized in the lattice. A shallow layer $(\approx 5 \mathrm{~nm})$ of implanted ${ }^{12} \mathrm{C}$ ions has been used as the source of vacancies for a deeper $(\approx 50 \mathrm{~nm}) \delta$-doped layer [49], taking advantage of annealing-induced diffusion. With this method, it is possible to increase the NV density in the $\delta$-doped layer by increasing the ${ }^{12} \mathrm{C}$ dose without activating $\mathrm{NV}$ centers in the substrate. An alternative method, applied to low energy helium implantation, consists of growing a thicker $\mathrm{N}$-doped layer $(d=18 \mathrm{~nm})$ and adding a last etching step to create NV centers near the surface [52].

NV yields reached values of 15\% [52] and 50\% [50], however, remaining quite low (1.9\%) in [49]. As discussed below, often, $\delta$-doping enhances the coherence times of shallow NVs compared to $\mathrm{N}$ implantation. Moreover, it potentially localizes NV centers in a more defined depth.

(a) Nitrogen $\delta$-doping

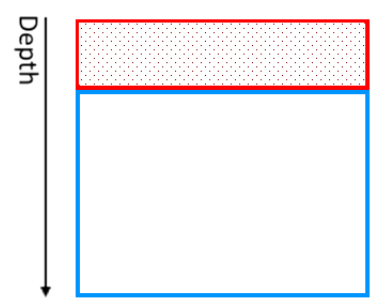

(b) Overgrowth of a diamond layer

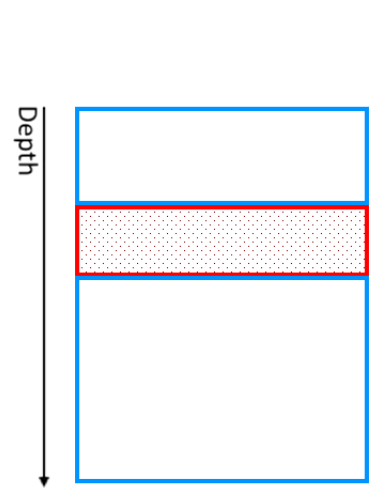

(c) Implantation/ irradiation +annealing

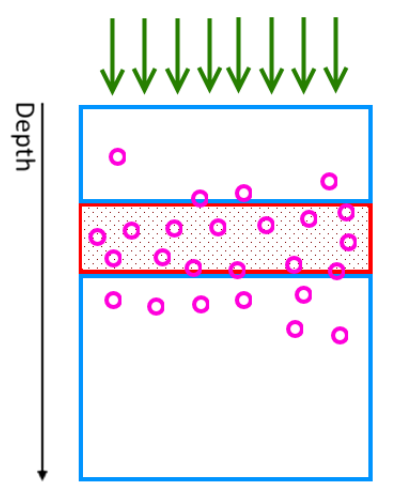

Figure 2. Schematic representation of the $\delta$-doping method. (a) Creation of an N-doped, several nm-thick layer, the $\delta$-doped layer (red, spotted rectangle). (b) Overgrowth with an undoped, epitaxial diamond layer (blue rectangle). (c) Implantation/irradiation and annealing. Pink circles indicate lattice vacancies close to the $\delta$-doped layer. 


\subsection{Photochromism, Quantum Efficiencies and Coherence Time}

$\mathrm{NV}$ centers exist in two luminescent charge states: negative $\left(\mathrm{NV}^{-}\right)$and neutral $\left(\mathrm{NV}^{0}\right)$ [54]; only $\mathrm{NV}^{-}$exhibits ODMR. To create an $\mathrm{NV}^{-}$, an electron has to be captured by the $\mathrm{NV}$ center. The $\mathrm{NV}^{-}$ population never exceeds 75-80\% (excitation wavelength 450-610 $\mathrm{nm}$ ) [55-57], due to photo-induced ionization [57]. In the band from $450-575 \mathrm{~nm}, \mathrm{NV}^{-}$and $\mathrm{NV}^{0}$ absorb light. Consequently, the loop of $\mathrm{NV}^{-}$excitation-ionization and $\mathrm{NV}^{0}$ excitation-recombination is closed, and the $\mathrm{NV}$ cycles between both charge states. The $\mathrm{NV}^{-}$steady state population is maximized for optical excitation in the band 510-540 nm [55]. Besides this photochromism, the NV charge state is directly influenced by the Fermi level position in diamond. For shallow NVs, the Fermi level position closely relates to the electron affinity of the surface (see Section 2.3).

An important characteristic for light-emitting systems is their quantum efficiency (QE). It is defined as $Q E=\frac{k_{r}}{\left(k_{r}+k_{n r}\right)}$, where $k_{r}, k_{n r}$ are the radiative and non-radiative decay rate of the NV center, respectively. A higher $\mathrm{QE}$, in general, leads to brighter emission from the centers. For shallow $\mathrm{NVs}$, QE can be as high as 70\% (82\%) for a depth of $4.1 \mathrm{~nm}(8 \mathrm{~nm})$, respectively [22]. The work in [22] estimates QE $>96 \%$ in bulk diamond. This result implies decreased QE for shallow NVs, which was attributed to non-radiative decays induced by surface strain. Generally, careful control of strain and crystalline quality seems necessary to obtain high QE: QE in the range of only $25-60 \%$ was found in ion-damaged diamond (H-implantation, fluence $F=1 \times 10^{15} \mathrm{~cm}^{-2}$ corresponding to an estimated induced vacancy density $d=60 \mathrm{ppm},[58])$. NV centers in 25-nm nanodiamonds even may show $\mathrm{QE}<20 \%$ [59].

Generally, shallowly-implanted NV centers show reduced $\mathrm{T}_{2}$ together with broadened ODMR lines $[16,41,60,61]$. Table 1 illustrates this reduction in comparison to deep, native centers. Degraded coherence properties are related to noise created by the proximity to the surface. The work in [60] presents a spectroscopic analysis of the noise $\left(\mathrm{N}\right.$ dose $10^{8} \mathrm{~cm}^{-2}$ ). High-frequency noise arises due to surface-modified phonons and low frequency noise due to the surface electronic spin bath. For ion doses of $10^{11} \mathrm{~cm}^{-2}$, noise produced by implanted defects close to NV centers adds to surface-related noise. Indeed, $\mathrm{N}$ bombardment introduces $\mathrm{N}$ impurities and vacancy complexes that act as paramagnetic centers, degrading the coherence time of NV centers [62,63].

Table 1. Typical value of $T_{2}$ for shallowly-implanted and native, bulk NV from [16]. Note that for centers at a depth of around $50 \mathrm{~nm}$, the coherence time of deep centers is restored [40].

\begin{tabular}{cccc}
\hline & Photocounts & $\mathrm{T}_{\mathbf{2}}(\mu \mathrm{s})$ & Linewidth (MHz) \\
\hline Very Shallow $\mathrm{NV}^{-}(2.1 \mathrm{~nm})$ & $5 \times 10^{4}$ & $12.2 \pm 0.6$ & 2 \\
Shallow $\mathrm{NV}^{-}(7.7 \mathrm{~nm})$ & $4 \times 10^{5}$ & $40.4 \pm 0.8$ & 1.2 \\
Native $\mathrm{NV}^{-}(6 \mu \mathrm{m})$ & $4 \times 10^{5}$ & $128 \pm 10$ & 1 \\
\hline
\end{tabular}

\subsection{Methods to Improve Stability and Photoluminescence}

The work in [44] shows that the $\mathrm{NV}^{-}$population is decreased at depths up to $200 \mathrm{~nm}$ compared to bulk. This effect is attributed to the presence of an electronic depletion layer at the etched diamond surface. In this depletion region, $\mathrm{N}$ donors cannot donate an electron to the NV center because they are ionized. Stabilizing the $\mathrm{NV}^{-}$state close to the surface involves controlling the Fermi level in diamond [64-67]. The Fermi level, and consequently the $\mathrm{NV}^{-}$population, can be controlled via chemical functionalization of the surface $[64,65,68,69]$ (see Figure $3 a, b$ ) by applying an electrolyte gate voltage [70] (see Figure 3c) using in-plane gate nanostructures [71] or via doping the diamond [67]. 
(a) Oxygenated

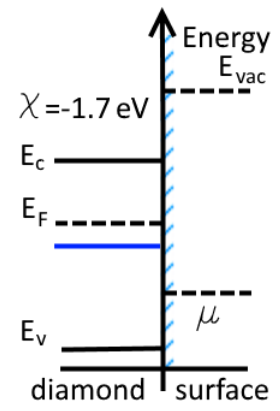

(b) Hydrogenated

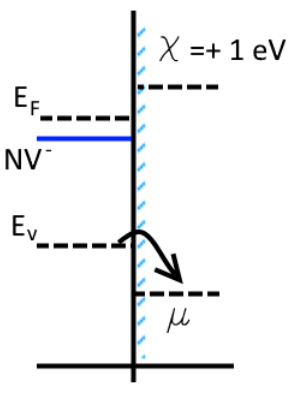

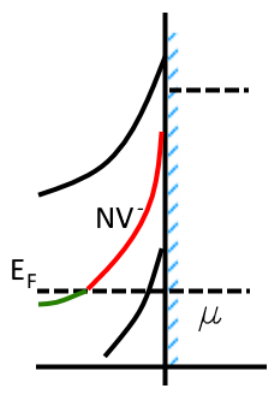

(c) Gate Voltage

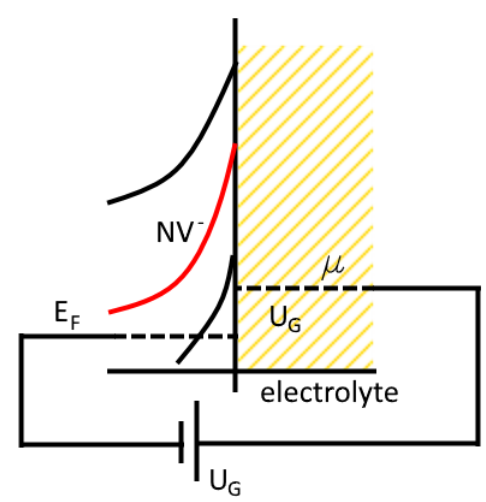

Figure 3. Energy band schematic of diamond energy levels for: (a) oxygenated surface; (b) hydrogenated surface. (c) Applying a gate voltage in the presence of an electrolyte.

First, we consider the decrease in the $\mathrm{NV}^{-}$population of shallow NV centers close to a hydrogen (H)-terminated surface compared to an oxygenated surface [64,65]. For H-terminated diamond, the conduction and valence bands shift upwards due to the negative electron affinity of the surface. The energy of the valence band maximum $\mathrm{E}_{V}$ is higher than the chemical potential $\mu$ of the electronic states created by adsorbed water. Electrons migrate to the adsorbate layer. In equilibrium, the diamond bands bend upwards; a 2D hole gas is created; and the $\mathrm{NV}^{-}$charge state is depleted [65]. Thus, $\mathrm{H}$-termination is not useful to stabilize $\mathrm{NV}^{-}$.

In contrast, fluorine (F)-terminated surfaces will lead to a downward bending of the diamond bands due to the large electron affinity of $\mathrm{F}$ [72]. In this case, the $\mathrm{NV}^{-}$population increases compared to H-terminated diamond [68] and also to oxygenated diamond [69]. However, it should be noted that so far, coherence measurements of shallow NV centers close to F-terminated surfaces are missing.

For H-terminated diamond, electrolytic gate electrodes can directly control the Fermi level [70] (see Figure 3c). An increase in the $\mathrm{NV}^{-}$ensemble population was observed for high implantation dose and positive gate voltage. Unfortunately, no single NV charge switching was observed, due to the high gate voltage needed for charge-state conversion at low implantation dose. On the other hand, this result was achieved in [71] by applying an electric field using in-plane nanostructures. The gate-voltage was applied to a structure formed by H-terminated areas and an O-terminated line, resulting in an offset between the Fermi level in the H-terminated areas on both sides of the line. In this way, switching the charge state of a single $\mathrm{NV}$ center from $\mathrm{NV}^{0}$ to $\mathrm{NV}^{-}$was established.

Deterministic electrical control of the charge state of a single NV center has been achieved using a $\mathrm{p}-\mathrm{i}-\mathrm{n}$ diode [66]. The NV center is positioned in the intrinsic region of the diode, and a current of holes is induced from the p-region. However, this technique converts $\mathrm{NV}$ centers to $\mathrm{NV}^{0}$. Using in plane Al-Schottky diodes, based on $\mathrm{H}$-terminated diamond, charge state switching of single $\mathrm{NV}$ centers $\left(\mathrm{NV}^{+}\right.$ to $\mathrm{NV}^{0}$ to $\mathrm{NV}^{-}$) has been obtained [73]. Implementing diode structures in scanning probe sensing seems, however, highly challenging.

Another way to change the Fermi level of diamond is by boron or phosphorus $(\mathrm{P})$ doping [67,74]. In particular, $\mathrm{P}$ impurities donate an electron to $\mathrm{NV}^{0}$, due to the fact that the $\mathrm{P}$ activation energy is low $\left(E_{A}=0.57 \mathrm{eV}\right)$ compared to NV acceptor states $\left(E_{N V}=2.58 \mathrm{eV}\right)$. In this way, a five-fold increase in luminescence and a pure $\mathrm{NV}^{-}$state was observed for a single $\mathrm{NV}$ [67] and a P doping $p=5 \times 10^{16} \mathrm{~cm}^{-3}$. It should be noted that this single $\mathrm{NV}$ had a short coherence time $\mathrm{T}_{2}=(19.77 \pm 0.27) \mu \mathrm{s}$, and the depth of the NV center in this case was not reported. 


\subsection{Methods to Improve $T_{2}$}

In this section, we discuss how $\mathrm{N} \delta$-doping $[47,51]$ and ion implantation $[52,75,76]$ or electron irradiation [50] can lead to improved spin coherence. Figure 4 summarizes recently obtained $\mathrm{T}_{2}$ values for different methods and works. As discussed above, implanted defects are sources of noise. Implanted defects can be of two types:

- Vacancy complexes [63];

- Ion impurities and crystal defects [77].

The formation of vacancy complexes during annealing is inhibited by Coulomb repulsion, if their charge state is changed from neutral to positive [63]. Vacancy charging is accomplished in a p-i junction by implanting $\mathrm{N}$ in the junction's space charge layer. A two-fold increase in yield and ten-fold increase in $T_{2}$ for very shallow NVs has been reported $\left(T_{2}=30 \mu \mathrm{s}, \mathrm{NV}\right.$ depth $\left.\mathrm{d}=3 \mathrm{~nm}\right)$.

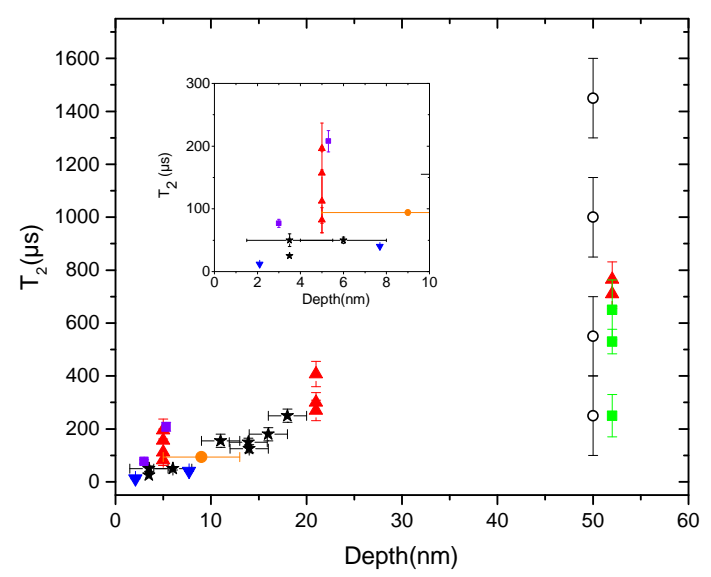

Helium atoms from De Oliveira et al. 2016

- Electrons from Ohno et al. 2012

- Electrons from McLellan et al. 2016

- Carbon atoms from Ohno et al. 2014

v Implanted $\mathrm{N}$ from Ofori-Okai et al. 2013

- Implanted N from Appel et al. 2016

- Implanted N and Oxidation from Lovchinsky et al. 2016 (Best and worse $\mathrm{T}_{2}$ values)

Figure 4. Coherence time $\mathrm{T}_{2}$ for $\mathrm{NV}$ centers created via $\delta$-doping methods and implantation with different inert species. For comparison, $\mathrm{T}_{2}$ of centers created via $\mathrm{N}$ implantation are given. The inset highlights $\mathrm{T}_{2}$ for an NV center less than $10 \mathrm{~nm}$ below the surface, which are especially important for sensing applications. Note the spread of $\mathrm{T}_{2}$ in the different works.

Noise from implantation-induced impurities and crystal defects can be reduced using $\delta$-doping and ex situ creation of vacancies (see Section 2.1). To be used in scanning probe sensing, NV centers should have a depth below $\approx 10 \mathrm{~nm}$. As mentioned before, this can be achieved by tuning the dimension of the cap layer [47] or adding a last etching step [52]. In this depth range, the maximum reached $\mathrm{T}_{2}$ is $200 \mu \mathrm{s}$ obtained when irradiating an isotopically-purified sample with electrons [47]. In this case, however, the NV density was too low for applications requiring the fabrication of single-crystal scanning probes, as discussed below.

We remark that NV areal densities around $D_{N V}=3 \times 10^{9} \mathrm{~cm}^{-2}$ are typically necessary for single crystal scanning probes: this corresponds to one NV center in a circular area with a diameter of $200 \mathrm{~nm}$ and thus to a single NV in typical nanostructures used for sensing (see Section 3). In [47], the $\delta$-doped layer has an $\mathrm{N}$ concentration of $C_{N}=3 \times 10^{16} \mathrm{~cm}^{-3}$, considering a layer thickness of $2 \mathrm{~nm}$; this results in an areal density of $\mathrm{N}$ of $6 \times 10^{9} \mathrm{~cm}^{-2}$, so in order to have an acceptable $\mathrm{NV}$ areal density, one should have a very high yield, around $50 \%$. A similar concentration of $\mathrm{N}\left(C_{N}=0.8 \pm 0.6 \times 10^{16} \mathrm{~cm}^{-3}\right)$ is reported in [49], leading to a low $\mathrm{NV}$ area density $\left(D_{N V}=10^{6} \mathrm{~cm}^{-2}\right)$. A slightly too low NV density is reported in [50] $D_{N V}=1.4 \times 10^{9} \mathrm{~cm}^{-2}$ and in [52] $D_{N V}=1 \times 10^{8} \mathrm{~cm}^{-2}$. It is worth noticing that an $\mathrm{N}$ volume concentration of $C_{N}=1.8 \times 10^{20} \mathrm{~cm}^{-3}$ is reported in [51]; this would result in an NV density of $D_{N V}=4 \times 10^{12} \mathrm{~cm}^{-2}$, considering a thickness layer of $2 \mathrm{~nm}$ and a yield of $10 \%$. In contrast, the $\mathrm{N}$ implantation method described in [63] seems more promising from this point of view: tuning the 
fluence of implanted $\mathrm{N}$ from $10^{10} \mathrm{~cm}^{-2}-10^{12} \mathrm{~cm}^{-2}(\mathrm{E}=5 \mathrm{keV})$ allows accessing the optimal densities of shallow NV centers.

We underline that till now, $\delta$-doping has not been applied in the manufacturing of diamond probes for nanosensing. This is probably due to the fact that $\delta$-doping involves the realization of sophisticated CVD methods. In contrast, creating single NVs by ion implantation can be achieved using commercial diamond material and implantation facilities.

Finally, we remark that surface oxidation has been used recently to improve $\mathrm{T}_{2}$ [78]. Wet oxidative chemistry and sample annealing at $465^{\circ} \mathrm{C}$ in a dry oxygen atmosphere increased $\mathrm{T}_{2}$ by an order of magnitude (max. $\mathrm{T}_{2}=208 \mu \mathrm{s}$ for a single NV, depth $(5.3 \pm 0.1) \mathrm{nm}, \mathrm{T}_{2}>100 \mu$ s observed for $6 \mathrm{NVs}$ ). Recent work [79], however, was not able to reproduce these results for shallower NVs: in [79], a similar oxidation procedure resulted in $\mathrm{T}_{2} \leq 6 \mu$ s for calculated depths of $(2.6 \pm 1.1) \mathrm{nm}$.

\section{Nanostructures for Photonics and Scanning Probe Operation}

NV centers close to the surface of bulk, single-crystal diamond are, on the one hand, advantageous as they reside in high-quality, high-purity, potentially low-stress diamond material mostly synthesized by the CVD method [80]. On the other hand, these NV centers do not fulfill all prerequisites for nanoscale sensing: first, positioning an NV created in a macroscopic diamond crystal within nanometer distance from a sample is hardly feasible. In principle, attaching the 'sample', e.g., the substance under investigation, to a scanning probe tip while keeping the NV center stationary can circumvent this issue. However, this limits the technique to microscopic samples, e.g., specific molecules or ions (e.g., [81]) for which attachment to a nano-sized tip is feasible.

Additionally, even for an optimal dipole orientation, a typical air microscope objective (NA 0.8 [82]) collects only about $5 \%$ of the fluorescence from an NV center in bulk diamond: as diamond exhibits a high refractive index of 2.4 for visible light [18], only light incident at angles $<24.6^{\circ}$ (partially) leaves the diamond as illustrated in Figure 5a. Other light rays are lost due to total internal reflection. Additionally, the dipolar emission from color centers is typically directed towards the optically-dense medium. Thus, in our case, emission is directed towards the bulk diamond, additionally hindering fluorescence detection $[83,84]$. Consequently, coupling to nanophotonic structures is mandatory to enhance the photon rates from individual color center sensors in single-crystal diamond. Alternatively, attaching nanodiamonds (NDs) containing NV centers to a scanning probe tip provides a scannable NV platform (e.g., [26,85]). However, NDs show non-ideal material properties, typically due to excess nitrogen or crystal strain resulting from milling of the material [86]. Thus, incorporated color centers may suffer from short coherence time, strong inhomogeneous line spreads and reduced stability [87]. In this review, we thus focus on single-crystal diamond-based sensing techniques.

Scanning probe sensing demands nanophotonic structures with several properties:

- Broadband operation: Section 1 and Figure 1c illustrate the $100 \mathrm{~nm}$-broad room temperature emission spectrum of NV centers. Room temperature spin read-out under non-resonant excitation is most efficient if the integral emission is detected. Thus, the photonic structure should allow for efficient fluorescence detection from the complete $\mathrm{NV}$ emission spectrum.

- Tip-like geometry and suitability for NV centers close to surfaces: a tip-like geometry ensures close proximity of the scannable NV center to the sample even in the presence of alignment uncertainties. The photonic structure needs to be functional for NV centers very close to diamond surfaces.

- Attachment to the feedback system: the nanostructure has to be attached to a force sensor, i.e., a tuning fork or a cantilever. A miniaturized structure is needed, thus avoiding strong shifting of the resonance frequency due to the additional mass or severe damping of the oscillating system.

Considering the first criterion, resonant photonic structures, like, e.g., photonic crystal cavities [88], which selectively enhance a specific transition within the emission spectrum, are not fully suitable for sensing applications under ambient conditions. For quantum information, in contrast, these systems 
are promising as they potentially form a coherent interface between spins and photons in quantum networks [89]. In our case, waveguide-like structures that channel the NV's broad emission into certain spatial modes and direct the light towards the collection optics (nanopillars; see Figure 5b) seem more suitable mainly due to their broadband operation. Certain waveguide-based structures, i.e., dielectric antennas [90], have been found to enable potentially near unity collection efficiency for NV fluorescence [91]. However, they consist of planar multilayer structures and are thus not optimized for scanning probe sensing. In contrast, they might be very suitable for approaches where a diamond chip is used for sensing (wide field approaches, lower spatial resolution, e.g., [92]). Taking into account the first two criteria, also solid immersion lenses, where color centers are buried inside diamond half spheres, are not suitable [93]. In this review, we focus on the nanostructure types suitable for scanning probe sensing. For more complete recent reviews of diamond nanophotonics in the context of quantum information, see [94-97].

(a)

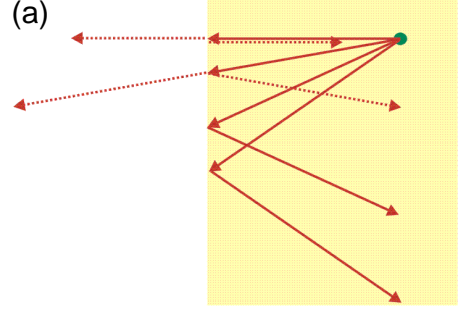

critical angle for total internal reflection at diamond-air interface $\alpha_{g}=24.6^{\circ}$

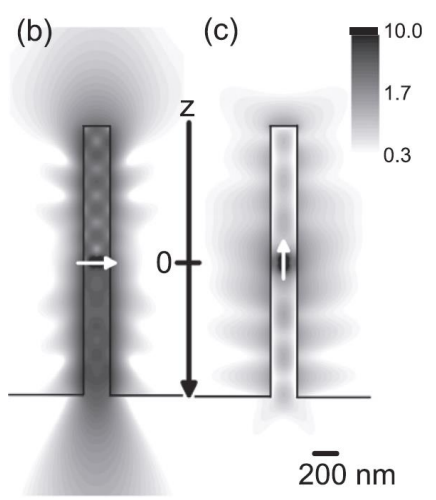

Figure 5. (a) Illustration of total internal reflection: light incident under low angles cannot leave the diamond; for normal incidence, roughly $17 \%$ of the incident power is reflected (Fresnel reflection). $(\mathbf{b}, \mathbf{c})$ These images show the magnitude of the electric field (simulated for a light wavelength of $\lambda=700 \mathrm{~nm}$ ) inside diamond nanopillars. Note that darker areas correspond to higher fields, as indicated in the color bar. The field is being channeled in the direction of the pillar axis, witnessing wave-guiding via the photonic modes of the pillars. Reprinted from [82] under CC-BY3.0. The arrow within the structures indicates the dipole orientation. For the optimal dipole orientation, where the dipole axis is perpendicular to the pillar axis, collection efficiencies up to $40 \%$ can be reached (collection into NA0.8 from the air side).

In essence, photonic nanostructures suitable for scanning probe sensing consist of a roughly tip-shaped nanophotonic structure on a thin (typically $<1 \mu \mathrm{m}$ ) diamond mounting structure, as shown in Figure 6a. First, operational sensors have been presented in 2012 [61]. The work in [41,98] presents more recent nanofabrication approaches. In a further very recent approach, mounting structures holding an array of pillars are used. Consequently, several NV centers are usable, and vector magnetometry might be feasible [99]. However, this approach introduces the risk that a pillar that is not in direct contact with the sample is used and, thus, an unwanted stand-off distance strongly reduces the resolution.

We start by addressing how large-area, thin, single-crystal diamond membranes can be formed. High-purity, CVD diamond with $<5$ ppb substitutional nitrogen and an intrinsically very low density of NV centers is commercially available (see Section 2.1). Starting from this material, thin, single-crystal diamond membranes can be formed by thinning down diamond plates obtained by polishing (typically several tens of $\mu \mathrm{m}$ thick). To thin the plates down, reactive-ion etching with different reactive gases in the plasma has been used among them chlorine and oxygen-based recipes [41,61], fully-chlorine-based chemistry [101], oxygen/fluorine-based chemistry [98,102] and fully-fluorine-based chemistry [7]. For all of these approaches except in [98], argon is added to the plasma to introduce physical etching (sputter etching) to the process. Alternatively, membranes can be created by damaging a buried layer 
of diamond via ion implantation. When this layer is graphitized upon annealing, it can be chemically etched, and membranes are lifted-off. However, due to strong ion damage, this material is not directly usable and has to be overgrown with a pristine layer of CVD diamond, adding technological complexity to the process [103]. We note that it is not possible to obtain thin, low-stress, single-crystal membranes directly via growth on a non-diamond substrate that might allow for wet-chemical etching of the substrate: for all diamond heteroepitaxy, the starting phase of the growth is highly defective, and forming high-quality, thin membranes includes removing this initial growth areas [104]. Reliable, large-area fabricating of single-crystal membranes is essential for high-yield fabrication of scanning probe devices and potential future up-scaling of the technology.

(a)

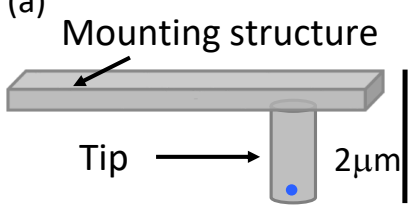

(b)

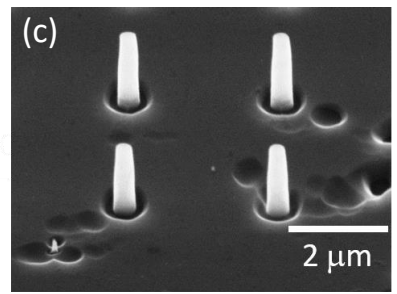

NV centers
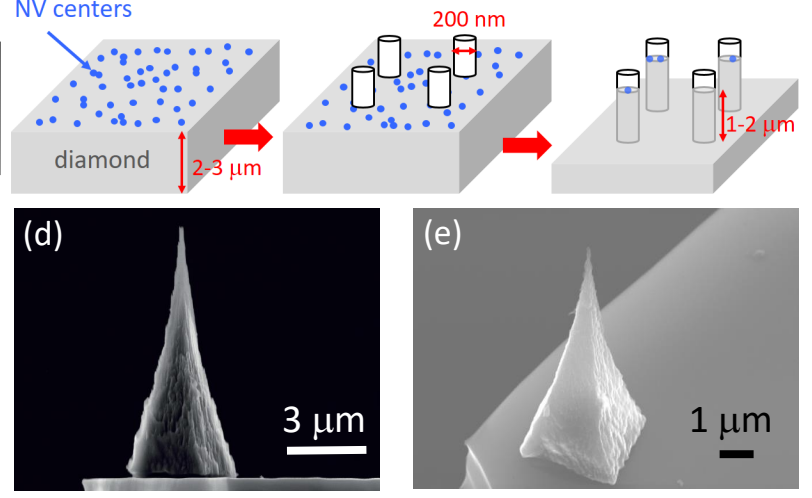

Figure 6. (a) Schematics of an all diamond scanning probe nanostructure, consisting of a cylindrical diamond tip on a thin mounting structure. (b) Illustration of the fabrication process of diamond pillars for sensing: shallowly-buried NV centers are created (blue dots: NV centers; grey: diamond). An etch mask (white) is created (typically via electron beam lithograph in Hydrogen silsesquioxane resist (here Dow Corning, Fox16)). The pillars are etched in a highly-anisotropic etch. NV centers not protected by the etch mask are removed with the surrounding diamond. If the optimal density is chosen, roughly one out of three pillars contains a single NV center [41]. (c) Electron microscopy image of diamond nanopillars etched into CVD diamond. (d,e) Diamond pyramids formed during a specialized CVD growth process and mounted to silicon cantilevers for scanning probe applications [100].

The nanostructure forming the tip is either almost cylindrical (termed nanopillar $[41,82,84,105,106])$, pyramidal [100] or features a truncated cone shape with a taper angle [84,107]. The work in [107] demonstrated that tapering the pillar enhances fluorescence rates from single NVs. Lithographically-defined etch masks are used to create the pillars, as illustrated in Figure 6b. These masks undergo faceting and erosion [108], thus limiting the pillar length and influencing its shape. For an electron microscopy image of diamond nanopillars, see Figure 6c. Mask erosion is especially critical, as a high-density plasma (mostly oxygen and argon) is needed to enable highly-anisotropic etching of diamond. For the coupling to the nanostructure's photonic modes, not only the placement of the NV center is essential, but also the orientation of the NV's electric dipoles. As indicated in Figure 5b, placing the NV's dipoles perpendicularly to the pillar axis is the most advantageous configuration. This situation can be reached in $<111>$-oriented diamond. Here, NV centers created during CVD growth preferentially align in the growth direction, with their dipoles in the plane perpendicular to that direction [109-111]. However, CVD growth in the $<111>$ crystal direction is challenging [112] and still leads to a material with lower quality than the standard $<100>$ growth. In $<100>$ diamond, NV centers align along all four equivalent $\langle 111\rangle$ directions and have an oblique angle of $54.7^{\circ}$ with the pillar axis. As an alternative, CVD growth in the $<113>$ direction has been investigated [113]. In this material, $73 \%$ of NVs form an angle of $29.5^{\circ}$ with the potential pillar axis and thus bring their dipoles closer to 
the ideal orientation. The work in [82] demonstrates pillars fabricated into $<111>$-oriented material. However, so far, optimized crystal orientations have not been used for scanning probe sensing.

To manufacture nanophotonic structures, two fundamentally different approaches exist: first, the structures can be sculpted from bulk diamond material (top-down approach) as discussed above $[41,82,84,105,108]$. In contrast, nanostructures might also be created directly during (CVD) synthesis of diamond (bottom up approach $[100,114,115]$ ). Pyramidal nanostructures formed via bottom-up approaches (see Figure 6d,e) also have advantageous photonic properties [100]. However, they currently show high levels of color center incorporation during growth. This results from growth on non-diamond materials or incorporation of impurities from masks used in the growth. Consequently, these structures are not usable for sensing with single color centers so far. In top-down approaches, plasma-based etching forms the nano-structures. The work in [116] shows that a plasma can damage the diamond surface and reduce NV coherence. From this point of view, bottom-up approaches are appealing as they offer the opportunity to avoid plasma etching of diamond.

As discussed in Section 2, shallow NV creation is a crucial step towards functional sensing devices. So far, NVs in nanopillars have been created by ion implantation $[41,61,106,107]$ followed by structuring the nanopillars via lithography and etching (see Figure 6b). Alternatively, NV centers created during growth, but at non-defined depth, were used [82,105]. Thus, the latter pillars are not suitable for scanning probe sensing. A promising alternative approach is spatially localized ion implantation into high-purity nanostructures. Three different approaches have been presented: the first uses focused nitrogen ion beams for localized implantation [117]. In the second approach, a pierced AFM tip is used as an implantation aperture [118]. In the third approach, single ion traps are planned to be used as deterministic sources of implanted ions [119]. Also in the context of $\delta$-doping, three-dimensional localization of NV centers has been reported: [49] uses a mask with nanometric apertures for single $\mathrm{NV}$ creation in a $\delta$-doped layer. For the smallest aperture of $(114 \pm 21) \mathrm{nm}$, single NVs were created in $18 \%$ of the apertures. In [50], a transmission electron microscope is used for irradiation, achieving sub-micron accuracy in lateral positioning.

We point out that in parallel to the efforts in nanophotonics, recently electrical read-out of the $\mathrm{NV}$ spin state has been proposed alternatively to the challenging optical read-out: NV centers are more likely to undergo multi-photon photo-ionization as long as they are not being shelved to the singlet states. Thus, an NV center in the $m_{s}=0$ spin state is more likely to be ionized and leads to a higher photo-current. Demonstrations include continuous [120], as well as pulsed detection [121]. Whereas the read-out contrast can approach $20 \%$, demonstrations of single NV read-out are still pending.

\section{Recent Advances in NV Sensing}

In this section, we review selected recent advances in sensing with single NV centers. In Section 4.1, we summarize recent steps towards scanning near-field optical microscopy (SNOM) with a single NV center. In Section 4.2, we present recent results using the magnetic sensing capabilities of NV centers (atomic size sensor, high sensitivity, low-invasiveness) to study physical phenomena at low temperature and/or few atoms level.

\subsection{Near-Field Microscopy with NV Centers}

In recent years, the highly-photostable emission of individual NV centers has triggered several efforts to realize near-field sensing based on NV centers $[8,26,122,123]$. In general, near-field-based imaging, where a sample is illuminated by the near-field of a light source, allows a higher resolution than far field-based techniques and is able to beat the Abbé limit of resolution.

Single NV centers were used as nano-sized light-source that is brought within $<100 \mathrm{~nm}$ of the sample in a SNOM setup $[25,123,124]$. A nanodiamond (ND) attached to the end of a SNOM tip achieved a resolution of around $50 \mathrm{~nm}$ when imaging metallic nanostructures, thus beating the Abbé limit of resolution (see Figure 7). The resolution was limited by the vertical NV-to-sample distance, 
which is constrained by the ND size and by the fact that the ND was not put in contact with the sample to avoid too strong friction forces at the tip apex.

Förster resonance energy transfer (FRET) was first described as non-radiative energy transfer between a pair of single molecules (dipoles) in close proximity [14,125]. To enable FRET, the emission band of the donor molecule has to overlap with the absorption band of the acceptor molecule, allowing energy transfer from the donor to the acceptor molecule. The transfer efficiency reaches $50 \%$ for a characteristic distance, the so-called Förster distance $R_{0}$, and drops with the inverse sixth power of the distance. NV centers in a FRET pair with a fluorescent dye have shown $\mathrm{R}_{0}=5.6 \mathrm{~nm}$ [126]. Thus, this effect promises truly nanoscale resolution in near-field-based imaging.

In recent experiments, an ND placed close to the apex of a commercial AFM tip was scanned close to a surface covered with graphene flakes [26]. The NV center and the graphene flake form a FRET pair. A Förster distance of $15 \mathrm{~nm}$ was found. Sekatskii and co-workers [122] reported on unsuccessful experiments on FRET transfer between a scanning NV and a dye molecule, despite previous demonstration of FRET to dye molecules covalently bonded to NDs [8]. This finding might relate to a varying quantum efficiency (QE) for NV centers in NDs [59], the need for accurate control of the ND surface (graphite layers), as well as to large stand-off distances when attaching an ND to a scanning probe tip. These issues may be addressable in the future using scanning probe devices sculpted out of single-crystal diamond [41,61]. So far, no experiments on near-field sensing based on single-crystal scanning probes have been reported.

Finally, we mention that super-resolution images of NV centers in bulk diamond were recorded using stimulated emission depletion microscopy [127], ground state depletion microscopy [128], stochastic optical reconstruction microscopy [129] and by sampling second- and third- order photon correlation function [130]. These techniques beat the Abbé resolution limit without the need for near-field imaging.

(a)
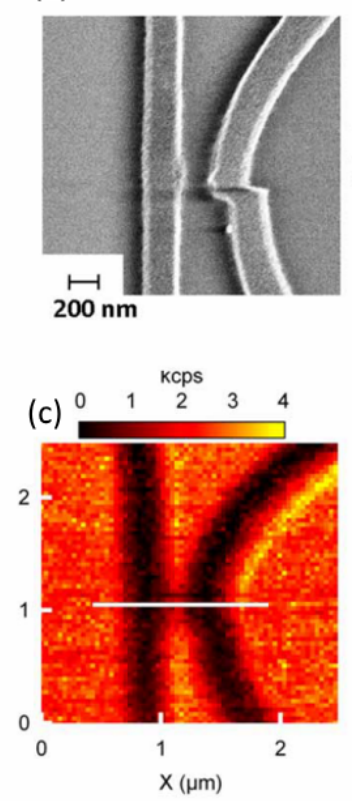

(d)

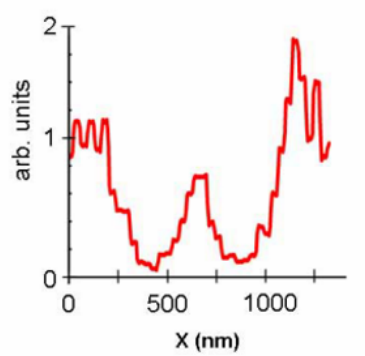

Figure 7. (a) Scanning electron micrograph of chromium structures patterned on a fused silica cover slip. (b) Numerically-flattened topography of the same region. (c) Fluorescence scanning near-field optical microscopy (SNOM) image recorded using the single photon tip. (d) cross-cut of the optical intensity along the white line in (c). Reprinted from [124], copyright the Optical Society of America (2009). 


\subsection{Recent Applications of Single NV Sensing}

The implementation of scanning NV-based microscopy at cryogenic temperatures forms a recent milestone: in a preliminary realization, cryogenic operation was achieved with a single NV center in bulk material and a scanning magnetic tip [131]. More recently, versatile approaches with NV centers in single crystal scanning probes have been presented $[99,132]$. This technology significantly broadens the range of samples for which NV magnetometry can be applied: solid materials present unique phenomena that only occur at cryogenic temperatures such as superconductivity. An ideal superconductor is supposed to expel all magnetic fields (Meissner effect). However, real superconductors present points at which magnetic fields penetrate and magnetic vortices form. NV nano-magnetometry was applied for a high-resolution, low-invasive imaging of superconducting vortices (6 K, [99], $4.2 \mathrm{~K}$ [132]). To achieve cryogenic operation, the NV microscope is enclosed in a liquid ${ }^{4} \mathrm{He}$ cryostat with optical access. Particular care was taken to avoid heating by microwave (MW) and laser excitation. Superconducting vortices were imaged with a resolution below $100 \mathrm{~nm}$ with magnetic field sensitivity of $30 \mu \mathrm{T} \cdot \mathrm{Hz}^{1 / 2}$ [99] and $11.9 \mu \mathrm{T} \cdot \mathrm{Hz}^{1 / 2}$ [132], allowing in [132] to verify vortex models beyond the monopole approximation.

Very recently, NV microscopy imaged nanoscale ferrimagnetic domains in antiferromagnetic random access memories [133]. We underline that part of these measurements were performed in zero field cooling (ZFC), taking advantage of low-invasive sensing using NV centers.

As discussed above, NV microscopy is often employed to sense static magnetic fields with nanoscale spatial resolution. However, NV centers are also sensitive to magnetic fields oscillating at $\mathrm{GHz}$ frequencies: single-crystal NV scanning probes imaged magnetic fields generated by MW currents with nanoscale resolution and sensitivity of a few $\mathrm{nA} \cdot \mathrm{Hz}^{1 / 2}$ [30]. The basic idea is to tune an NV spin resonance to the investigated MW frequency using a static magnetic field. The induced coherent spin oscillation frequency (Rabi frequency) then maps the magnetic field amplitude.

Single NV centers also form atomic-sized probes for nuclear magnetic resonance (NMR) signals [134-136]. The NV spin state is sensitive to the stochastic transverse magnetization at the Larmor frequency of the investigated ensemble of nuclei. Using suitable pulse sequences, it is possible to filter NMR signals and recover NMR spectra. Using NV probes, NMR investigations of small ensembles of nuclei are feasible. In contrast, conventional NMR spectroscopy is limited to macroscopic, thermally-polarized ensembles in high fields. Recent work presents NMR studies of a single protein [78] and of atomically-thin hexagonal boron nitride (h-BN) layers [137]. ${ }^{2} \mathrm{H}$ and ${ }^{13} \mathrm{C} N \mathrm{MR}$ spectra of ubiquitin proteins were measured [78], paving the way for experimental studies of biological systems at the single-molecule level. Conventional nuclear quadrupole resonance (NQR) [138] spectroscopy yields important information on chemical properties of macroscopic samples. However, it suffers from poor sensitivity due to low thermal polarization. This issue can be overcome using single NV centers that are sensitive to stochastic nuclear magnetization. The stochastic polarization is proportional to $\sqrt{n}$, where $n$ is the number of nuclei, but does not depend on the applied field. Single NV NQR was applied to study thin h-BN flakes, verifying a correlation between the number of atomic layers and a shift in NQR spectra [137].

NMR imaging has already been partially combined with scanning NV microscopy, in the sense that samples on a tip were scanned above a stationary NV. This configuration has been used for magnetic resonance imaging of ${ }^{1} \mathrm{H}$ nuclei with a spatial resolution of $12 \mathrm{~nm}$ [139]. ${ }^{19} \mathrm{~F}$ nuclei were imaged in a calibration grating [140]; widths of $(29 \pm 2) \mathrm{nm}$ were resolved [140]. To the best of our knowledge, to date, there are no reports on the use of single-crystal diamond scanning probes for NMR imaging. However, this fact does not represent a general limitation. It most probably relates to the fact that because of challenging nanofabrication procedures, only a few research groups have access to single-crystal scanning probes so far. 


\section{Conclusions}

We have summarized recent achievements and the basics of NV center-based sensing. The fabrication of suitable photonic nanostructures that allow scanning probe sensing and high photon rates from single centers, as well as the creation of NV color centers close to the diamond surface are still challenging subjects of research. To date, many recent advances like the creation of NV centers via $\delta$-doping methods, optimized surface treatments and doping of diamond, as well as diamond crystals with optimized orientation have not yet been used in the challenging fabrication of single-crystal diamond scanning probes. Thus, enhanced sensitivities and resolution in NV-based imaging are feasible in the future, given that the recent advances in material sciences are fully transferred to the sensor fabrication. As discussed in this review, many approaches to optimal, shallow NV centers are investigated. The results considering creation yield and NV properties are not always fully consistent, illustrating the complexity of the field. Moreover, the field could tremendously profit from simplified procedures to upscale the fabrication of optimized sensing nanostructures.

Acknowledgments: The authors acknowledge funding via a NanoMatFutur grant of the German Ministry of Education and Research. Elke Neu acknowledges funding via a PostDoc fellowship of the Daimler and Benz foundation.

Author Contributions: Ettore Bernardi and Elke Neu wrote the manuscript. Selda Sonusen and Richard Nelz performed the experiments and contributed experimental data (NV spectroscopy, ODMR, nanowires). All authors discussed and commented on the manuscript.

Conflicts of Interest: The authors declare no conflict of interest.

\section{References}

1. Dresselhaus, M.S. A revolution of nanoscale dimensions. Nat. Rev. Mater. 2016, 1, 15017.

2. Wilhelm, S.; Tavares, A.J.; Dai, Q.; Ohta, S.; Audet, J.; Dvorak, H.F.; Chan, W.C. Analysis of nanoparticle delivery to tumours. Nat. Rev. Mater. 2016, 1, 16014.

3. Wagner, T.; Strasberg, P.; Bayer, J.C.; Rugeramigabo, E.P.; Brandes, T.; Haug, R.J. Strong suppression of shot noise in a feedback-controlled single-electron transistor. Nat. Nanotechnol. 2017, 12, 218-222.

4. Nowodzinski, A.; Chipaux, M.; Toraille, L.; Jacques, V.; Roch, J.F.; Debuisschert, T. Nitrogen-Vacancy centers in diamond for current imaging at the redistributive layer level of Integrated Circuits. Microelectron. Reliab. 2015, 55, 1549-1553.

5. Dolde, F.; Doherty, M.W.; Michl, J.; Jakobi, I.; Naydenov, B.; Pezzagna, S.; Meijer, J.; Neumann, P.; Jelezko, F.; Manson, N.B.; et al. Nanoscale Detection of a Single Fundamental Charge in Ambient Conditions Using the $\mathrm{NV}^{-}$Center in Diamond. Phys. Rev. Lett. 2014, 112, 097603.

6. Kucsko, G.; Maurer, P.; Yao, N.; Kubo, M.; Noh, H.; Lo, P.; Park, H.; Lukin, M. Nanometre-scale thermometry in a living cell. Nature 2013, 500, 54-58.

7. Ali Momenzadeh, S.; de Oliveira, F.F.; Neumann, P.; Bhaktavatsala Rao, D.D.; Denisenko, A.; Amjadi, M.; Chu, Z.; Yang, S.; Manson, N.B.; Doherty, M.W.; et al. Thin Circular Diamond Membrane with Embedded Nitrogen-Vacancy Centers for Hybrid Spin-Mechanical Quantum Systems. Phys. Rev. Appl. 2016, 6, 024026.

8. Tisler, J.; Reuter, R.; Lämmle, A.; Jelezko, F.; Balasubramanian, G.; Hemmer, P.R.; Reinhard, F.; Wrachtrup, J. Highly Efficient FRET from a Single Nitrogen-Vacancy Center in Nanodiamonds to a Single Organic Molecule. ACS Nano 2011, 5, 7893-7898.

9. Binnig, G.; Quate, C.F.; Gerber, C. Atomic Force Microscope. Phys. Rev. Lett. 1986, 56, 930-933.

10. Martin, Y.; Wickramasinghe, H.K. Magnetic imaging by force microscopy with $1000 \AA$ resolution. Appl. Phys. Lett. 1987, 50, 1455-1457.

11. Ohnesorge, F.; Binnig, G. True Atomic Resolution by Atomic Force Microscopy Through Repulsive and Attractive Forces. Science 1993, 260, 1451-1456.

12. Grinolds, M.; Hong, S.; Maletinsky, P.; Luan, L.; Lukin, M.; Walsworth, R.; Yacoby, A. Nanoscale magnetic imaging of a single electron spin under ambient conditions. Nat. Phys. 2013, 9, 2145.

13. Rondin, L.; Tetienne, J.P.; Hingant, T.; Roch, J.F.; Maletinsky, P.; Jacques, V. Magnetometry with nitrogen-vacancy defects in diamond. Rep. Prog. Phys. 2014, 77, 056503.

14. Förster, T. Zwischenmolekulare Energiewanderung und Fluoreszenz. Ann. Phys. 1948, 437, 55-75. 
15. Acosta, V.; Hemmer, P. Nitrogen-vacancy centers: Physics and applications. MRS Bull. 2013, 38, 127-130.

16. Ofori-Okai, B.K.; Pezzagna, S.; Chang, K.; Loretz, M.; Schirhagl, R.; Tao, Y.; Moores, B.A.; Groot-Berning, K.; Meijer, J.; Degen, C.L. Spin properties of very shallow nitrogen vacancy defects in diamond. Phys. Rev. B 2012, 86, 081406.

17. Bradac, C.; Gaebel, T.; Pakes, C.I.; Say, J.M.; Zvyagin, A.V.; Rabeau, J.R. Effect of the Nanodiamond Host on a Nitrogen-Vacancy Color-Centre Emission State. Small 2013, 9, 132-139.

18. Zaitsev, A. Optical Properties of Diamond: A Data Handbook; Springer: Berlin/Heidelberg, Germany, 2001.

19. Aharonovich, I.; Castelletto, S.; Simpson, D.; Su, C.; Greentree, A.; Prawer, S. Diamond-based single-photon emitters. Rep. Prog. Phys. 2011, 74, 076501.

20. Kraus, H.; Soltamov, V.; Fuchs, F.; Simin, D.; Sperlich, A.; Baranov, P.; Astakhov, G.; Dyakonov, V. Magnetic field and temperature sensing with atomic-scale spin defects in silicon carbide. Sci. Rep. 2014, 4, 5303.

21. Davies, G.; Hamer, M. Optical studies of the $1.945 \mathrm{eV}$ vibronic band in diamond. Philos. Trans. Roy. Soc. A 1976, 348, 285.

22. Radko, I.P.; Boll, M.; Israelsen, N.M.; Raatz, N.; Meijer, J.; Jelezko, F.; Andersen, U.L.; Huck, A. Determining the internal quantum efficiency of shallow-implanted nitrogen-vacancy defects in bulk diamond. Opt. Express 2016, 24, 27715-27725.

23. Alegre, T.P.M.; Santori, C.; Medeiros-Ribeiro, G.; Beausoleil, R.G. Polarization-selective excitation of nitrogen vacancy centers in diamond. Phys. Rev. B 2007, 76, 165205.

24. Kurtsiefer, C.; Mayer, S.; Zarda, P.; Weinfurter, H. Stable Solid-State Source of Single Photons. Phys. Rev. Lett. 2000, 85, 290-293.

25. Kühn, S.; Hettich, C.; Schmitt, C.; Poizat, J.P.; Sandoghdar, V. Diamond colour centres as a nanoscopic light source for scanning near-field optical microscopy. J. Microsc. 2001, 202, 2-6.

26. Tisler, J.; Oeckinghaus, T.; Stöhr, R.J.; Kolesov, R.; Reuter, R.; Reinhard, F.; Wrachtrup, J. Single defect center scanning near-field optical microscopy on graphene. Nano Lett. 2013, 13, 3152-3156.

27. Manson, N.B.; Harrison, J.P.; Sellars, M.J. Nitrogen-vacancy center in diamond: Model of the electronic structure and associated dynamics. Phys. Rev. B 2006, 74, 104303.

28. Collins, A.; Thomaz, M.; Jorge, M. Luminescence decay time of the $1.945 \mathrm{eV}$ centre in type Ib diamond. J. Phys. C 1983, 16, 2177-2181.

29. Robledo, L.; Bernien, H.; van der Sar, T.; Hanson, R. Spin dynamics in the optical cycle of single nitrogen-vacancy centres in diamond. New J. Phys. 2011, 13, 025013.

30. Appel, P.; Ganzhorn, M.; Neu, E.; Maletinsky, P. Nanoscale microwave imaging with a single electron spin in diamond. New J. Phys. 2015, 17, 112001.

31. Dréau, A.; Lesik, M.; Rondin, L.; Spinicelli, P.; Arcizet, O.; Roch, J.F.; Jacques, V. Avoiding power broadening in optically detected magnetic resonance of single NV defects for enhanced dc magnetic field sensitivity. Phys. Rev. B 2011, 84, 195204.

32. Gruber, A.; Dräbenstedt, A.; Tietz, C.; Fleury, L.; Wrachtrup, J.; von Borczyskowski, C. Scanning confocal optical microscopy and magnetic resonance on single defect centers. Science 1997, 276, 2012-2014.

33. Balasubramanian, G.; Neumann, P.; Twitchen, D.; Markham, M.; Kolesov, R.; Mizuochi, N.; Isoya, J.; Achard, J.; Beck, J.; Tissler, J.; et al. Ultralong spin coherence time in isotopically engineered diamond. Nat. Mater. 2009, 8, 383-387.

34. Ermakova, A.; Pramanik, G.; Cai, J.M.; Algara-Siller, G.; Kaiser, U.; Weil, T.; Tzeng, Y.K.; Chang, H.C.; McGuinness, L.P.; Plenio, M.B.; et al. Detection of a Few Metallo-Protein Molecules Using Color Centers in Nanodiamonds. Nano Lett. 2013, 13, 3305-3309.

35. Degen, C.; Reinhard, F.; Cappellaro, P. Quantum sensing. arXiv 2016, arXiv:1611.02427.

36. Robledo, L.; Childress, L.; Bernien, H.; Hensen, B.; Alkemade, P.F.; Hanson, R. High-fidelity projective read-out of a solid-state spin quantum register. Nature 2011, 477, 574-578.

37. Luan, L.; Grinolds, M.S.; Hong, S.; Maletinsky, P.; Walsworth, R.L.; Yacoby, A. Decoherence imaging of spin ensembles using a scanning single-electron spin in diamond. Sci. Rep. 2015, 5, 8119.

38. Ryan, C.A.; Hodges, J.S.; Cory, D.G. Robust Decoupling Techniques to Extend Quantum Coherence in Diamond. Phys. Rev. Lett. 2010, 105, 200402.

39. Ziegler, J.F.; Ziegler, M.D.; Biersack, J.P. SRIM-The stopping and range of ions in matter (2010). Nucl. Instrum. Methods Phys. Res. Sect. B Beam Interact. Mater. At. 2010, 268, 1818-1823. 
40. Wang, J.; Zhang, W.; Zhang, J.; You, J.; Li, Y.; Guo, G.; Feng, F.; Song, X.; Lou, L.; Zhua, W.; Wang, G. Coherence times of precise depth controlled NV centers in diamond. Nanoscale 2016, 8, 5780-5785.

41. Appel, P.; Neu, E.; Ganzhorn, M.; Barfuss, A.; Batzer, M.; Gratz, M.; Tschöpe, A.; Maletinsky, P. Fabrication of all diamond scanning probes for nanoscale magnetometry. Rev. Sci. Instrum. 2016, 87, 063703.

42. Antonov, D.; Häußermann, T.; Aird, A.; Roth, J.; Trebin, H.R.; Müller, C.; McGuinness, L.; Jelezko, F.; Yamamoto, T.; Isoya, J.; et al. Statistical investigations on nitrogen-vacancy center creation. Appl. Phys. Lett. 2014, 104, 012105.

43. Orwa, J.O.; Santori, C.; Fu, K.M.C.; Gibson, B.; Simpson, D.; Aharonovich, I.; Stacey, A.; Cimmino, A.; Balog, P.; Markham, M.; et al. Engineering of nitrogen-vacancy color centers in high purity diamond by ion implantation and annealing. J. Appl. Phys. 2011, 109, 083530.

44. Santori, C.; Barclay, P.E.; Fu, K.M.C.; Beausoleil, R.G. Vertical distribution of nitrogen-vacancy centers in diamond formed by ion implantation and annealing. Phys. Rev. B 2009, 79, 125313.

45. Pezzagna, S.; Naydenov, B.; Jelezko, F.; Wrachtrup, J.; Meijer, J. Creation efficiency of nitrogen-vacancy centres in diamond. New J. Phys. 2010, 12, 065017.

46. Fávaro de Oliveira, F.; Momenzadeh, S.A.; Antonov, D.; Fedder, H.; Denisenko, A.; Wrachtrup, J. On the efficiency of combined ion implantation for the creation of near-surface nitrogen-vacancy centers in diamond. Phys. Status Solidi A 2016, 213, 2044-2050.

47. Ohno, K.; Heremans, F.J.; Bassett, L.C.; Myers, B.A.; Toyli, D.M.; Jayich, A.C.B.; Palmstrom, C.J.; Awschalom, D.D. Engineering shallow spins in diamond with nitrogen delta-doping. Appl. Phys. Lett. 2012, 101, 082413.

48. Osterkamp, C.; Lang, J.; Scharpf, J.; Müller, C.; McGuinness, L.P.; Diemant, T.; Behm, R.J.; Naydenov, B.; Jelezko, F. Stabilizing shallow color centers in diamond created by nitrogen delta-doping using SF6 plasma treatment. Appl. Phys. Lett. 2015, 106, 113109.

49. Ohno, K.; Joseph Heremans, F.; de las Casas, C.F.; Myers, B.A.; Alemán, B.J.; Bleszynski Jayich, A.C.; Awschalom, D.D. Three-dimensional localization of spins in diamond using ${ }^{12} \mathrm{C}$ implantation. Appl. Phys. Lett. 2014, 105, 052406.

50. McLellan, C.A.; Myers, B.A.; Kraemer, S.; Ohno, K.; Awschalom, D.D.; Bleszynski Jayich, A.C. Patterned formation of highly coherent nitrogen-vacancy centers using a focused electron irradiation technique. Nano Lett. 2016, 16, 2450-2454.

51. Chandran, M.; Michaelson, S.; Saguy, C.; Hoffman, A. Fabrication of a nanometer thick nitrogen delta doped layer at the sub-surface region of (100) diamond. Appl. Phys. Lett. 2016, 109, 221602.

52. Favaro de Oliveira, F.; Momenzadeh, S.A.; Antonov, D.; Scharpf, J.; Osterkamp, C.; Naydenov, B.; Jelezko, F.; Denisenko, A.; Wrachtrup, J. Toward Optimized Surface $\delta$-Profiles of Nitrogen-Vacancy Centers Activated by Helium Irradiation in Diamond. Nano Lett. 2016, 16, 2228-2233.

53. Kleinsasser, E.E.; Stanfield, M.M.; Banks, J.K.Q.; Zhu, Z.; Li, W.D.; Acosta, V.M.; Watanabe, H.; Itoh, K.M.; $\mathrm{Fu}$, K.M.C. High density nitrogen-vacancy sensing surface created via $\mathrm{He}+$ ion implantation of C-12 diamond. Appl. Phys. Lett. 2016, 108, 202401.

54. Mita, Y. Change of absorption spectra in type-Ib diamond with heavy neutron irradiation. Phys. Rev. B 1996, 53, 11360-11364.

55. Aslam, N.; Waldherr, G.; Neumann, P.; Jelezko, F.; Wrachtrup, J. Photo-induced ionization dynamics of the nitrogen vacancy defect in diamond investigated by single-shot charge state detection. New J. Phys. 2013, 15, 013064.

56. Chen, X.D.; Sun, F.W.; Zou, C.L.; Cui, J.M.; Zhou, L.M.; Guo, G.C. Vector magnetic field sensing by a single nitrogen vacancy center in diamond. EPL 2013, 101, 67003.

57. Beha, K.; Batalov, A.; Manson, N.B.; Bratschitsch, R.; Leitenstorfer, A. Optimum Photoluminescence Excitation and Recharging Cycle of Single Nitrogen-Vacancy Centers in Ultrapure Diamond. Phys. Rev. Lett. 2012, 109, 097404.

58. Gatto Monticone, D.; Quercioli, F.; Mercatelli, R.; Soria, S.; Borini, S.; Poli, T.; Vannoni, M.; Vittone, E.; Olivero, P. Systematic study of defect-related quenching of NV luminescence in diamond with time-correlated single-photon counting spectroscopy. Phys. Rev. B 2013, 88, 155201.

59. Mohtashami, A.; Koenderink, A.F. Suitability of nanodiamond nitrogen-Vacancy centers for spontaneous emission control experiments. New J. Phys. 2013, 15, 043017. 
60. Romach, Y.; Müller, C.; Unden, T.; Rogers, L.J.; Isoda, T.; Itoh, K.M.; Markham, M.; Stacey, A.; Meijer, J.; Pezzagna, S.; et al. Spectroscopy of Surface-Induced Noise Using Shallow Spins in Diamond. Phys. Rev. Lett. 2015, 114, 017601.

61. Maletinsky, P.; Hong, S.; Grinolds, M.; Hausmann, B.; Lukin, M.; Walsworth, R.; Loncar, M.; Yacoby, A. A robust scanning diamond sensor for nanoscale imaging with single nitrogen-vacancy centres. Nat. Nanotechnol. 2012, 7, 320-324.

62. Yamamoto, T.; Umeda, T.; Watanabe, K.; Onoda, S.; Markham, M.L.; Twitchen, D.J.; Naydenov, B.; McGuinness, L.P.; Teraji, T.; Koizumi, S.; Dolde, F.; et al. Extending spin coherence times of diamond qubits by high-temperature annealing. Phys. Rev. B 2013, 88, 075206.

63. de Oliveira, F.F.; Antonov, D.; Wang, Y.; Neumann, P.; Momenzadeh, S.A.; Häußermann, T.; Pasquarelli, A.; Denisenko, A.; Wrachtrup, J. Tailoring spin defects in diamond. arXiv 2017, arXiv:1701.07055.

64. Fu, K.M.C.; Santori, C.; Barclay, P.E.; Beausoleil, R.G. Conversion of neutral nitrogen-vacancy centers to negatively charged nitrogen-vacancy centers through selective oxidation. Appl. Phys. Lett. 2010, 96, 121907.

65. Hauf, M.V.; Grotz, B.; Naydenov, B.; Dankerl, M.; Pezzagna, S.; Meijer, J.; Jelezko, F.; Wrachtrup, J.; Stutzmann, M.; Reinhard, F.; et al. Chemical control of the charge state of nitrogen-vacancy centers in diamond. Phys. Rev. B 2011, 83, 081304(R).

66. Doi, Y.; Makino, T.; Kato, H.; Takeuchi, D.; Ogura, M.; Okushi, H.; Morishita, H.; Tashima, T.; Miwa, S.; Yamasaki, S.; et al. Deterministic Electrical Charge-State Initialization of Single Nitrogen-Vacancy Center in Diamond. Phys. Rev. X 2014, 4, 011057.

67. Doi, Y.; Fukui, T.; Kato, H.; Makino, T.; Yamasaki, S.; Tashima, T.; Morishita, H.; Miwa, S.; Jelezko, F.; Suzuki, Y.; et al. Pure negatively charged state of the NV center in $n$-type diamond. Phys. Rev. $B$ 2016, 93, 081203.

68. Shanley, T.W.; Martin, A.A.; Aharonovich, I.; Toth, M. Localized chemical switching of the charge state of nitrogen-vacancy luminescence centers in diamond. Appl. Phys. Lett. 2014, 105, 063103.

69. Cui, S.; Hu, E.L. Increased negatively charged nitrogen-vacancy centers in fluorinated diamond. Appl. Phys. Lett. 2013, 103, 051603.

70. Grotz, B.; Hauf, M.V.; Dankerl, M.; Naydenov, B.; Pezzagna, S.; Meijer, J.; Jelezko, F.; Wrachtrup, J.; Stutzmann, M.; Reinhard, F.; et al. Charge state manipulation of qubits in diamond. Nat. Commun. 2012, 3, 729.

71. Hauf, M.V.; Simon, P.; Aslam, N.; Pfender, M.; Neumann, P.; Pezzagna, S.; Meijer, J.; Wrachtrup, J.; Stutzmann, M.; Reinhard, F.; et al. Addressing single nitrogen-vacancy centers in diamond with transparent in-plane gate structures. Nano Lett. 2014, 14, 2359-2364.

72. Rietwyk, K.J.; Wong, S.L.; Cao, L.; O’Donnell, K.M.; Ley, L.; Wee, A.T.S.; Pakes, C.I. Work function and electron affinity of the fluorine-terminated (100) diamond surface. Appl. Phys. Lett. 2013, 102, 091604.

73. Schreyvogel, C.; Polyakov, V.; Wunderlich, R.; Meijer, J.; Nebel, C.E. Active charge state control of single NV centres in diamond by in-plane Al-Schottky junctions. Sci. Rep. 2015, 5, 12160.

74. Groot-Berning, K.; Raatz, N.; Dobrinets, I.; Lesik, M.; Spinicelli, P.; Tallaire, A.; Achard, J.; Jacques, V.; Roch, J.F.; Zaitsev, A.M.; et al. Passive charge state control of nitrogen-vacancy centres in diamond using phosphorous and boron doping. Phys. Status Solidi A 2014, 211, 2268-2273.

75. Huang, Z.; Li, W.D.; Santori, C.; Acosta, V.M.; Faraon, A.; Ishikawa, T.; Wu, W.; Winston, D.; Williams, R.S.; Beausoleil, R.G. Diamond nitrogen-vacancy centers created by scanning focused helium ion beam and annealing. Appl. Phys. Lett. 2013, 103, 081906.

76. Naydenov, B.; Richter, V.; Beck, J.; Steiner, M.; Neumann, P.; Balasubramanian, G.; Achard, J.; Jelezko, F.; Wrachtrup, J.; Kalish, R. Enhanced generation of single optically active spins in diamond by ion implantation. Appl. Phys. Lett. 2010, 96, 163108.

77. Wang, P.; Ju, C.; Shi, F.; Du, J. Optimizing ultrasensitive single electron magnetometer based on nitrogen-vacancy center in diamond. Chin. Sci. Bull. 2013, 58, 2920-2923.

78. Lovchinsky, I.; Sushkov, A.; Urbach, E.; de Leon, N.; Choi, S.; De Greve, K.; Evans, R.; Gertner, R.; Bersin, E.; Müller, C.; et al. Nuclear magnetic resonance detection and spectroscopy of single proteins using quantum logic. Science 2016, 351, 836-841.

79. Yamano, H.; Kawai, S.; Kato, K.; Kageura, T.; Inaba, M.; Okada, T.; Higashimata, I.; Haruyama, M.; Tanii, T.; Yamada, K.; et al. Charge state stabilization of shallow nitrogen vacancy centers in diamond by oxygen surface modification. Jpn. J. Appl. Phys. 2017, 56, 04CK08. 
80. Balmer, R.S.; Brandon, J.R.; Clewes, S.L.; Dhillon, H.K.; Dodson, J.M.; Friel, I.; Inglis, P.N.; Madgwick, T.D.; Markham, M.L.; Mollart, T.P.; et al. Chemical vapour deposition synthetic diamond: Materials, technology and applications. J. Phys. Condens. Matter 2009, 21, 364221.

81. Pelliccione, M.; Myers, B.A.; Pascal, L.M.A.; Das, A.; Bleszynski Jayich, A.C. Two-Dimensional Nanoscale Imaging of Gadolinium Spins via Scanning Probe Relaxometry with a Single Spin in Diamond. Phys. Rev. Appl. 2014, 2, 054014.

82. Neu, E.; Appel, P.; Ganzhorn, M.; Miguel-Sanchez, J.; Lesik, M.; Mille, V.; Jacques, V.; Tallaire, A.; Achard, J.; Maletinsky, P. Photonic nano-structures on (111)-oriented diamond. Appl. Phys. Lett. 2014, 104, 153108.

83. Lukosz, W.; Kunz, R. Light-emission By Magnetic And Electric Dipoles Close To A Plane Interface. 1. Total Radiated Power. J. Opt. Soc. Am. 1977, 67, 1607-1615.

84. Hausmann, B.J.; Khan, M.; Zhang, Y.; Babinec, T.M.; Martinick, K.; McCutcheon, M.; Hemmer, P.R.; Loncar, M. Fabrication of diamond nanowires for quantum information processing applications. Diam. Relat. Mater. 2010, 19, 621-629.

85. Tetienne, J.P.; Hingant, T.; Martinez, L.; Rohart, S.; Thiaville, A.; Diez, L.H.; Garcia, K.; Adam, J.P.; Kim, J.V.; Roch, J.F.; et al. The nature of domain walls in ultrathin ferromagnets revealed by scanning nanomagnetometry. Nat. Commun. 2015, 6, 6733.

86. Santori, C.; Barclay, P.E.; Fu, K.M.C.; Beausoleil, R.G.; Spillane, S.; Fisch, M. Nanophotonics for quantum optics using nitrogen-vacancy centers in diamond. Nanotechnology 2010, 21, 274008.

87. Bradac, C.; Gaebel, T.; Naidoo, N.; Sellars, M.J.; Twamley, J.; Brown, L.J.; Barnard, A.S.; Plakhotnik, T.; Zvyagin, A.V.; Rabeau, J.R. Observation and control of blinking nitrogen-vacancy centres in discrete nanodiamonds. Nat. Nanotechnol. 2010, 5, 345-349.

88. Faraon, A.; Santori, C.; Huang, Z.; Acosta, V.M.; Beausoleil, R.G. Coupling of Nitrogen-Vacancy Centers to Photonic Crystal Cavities in Monocrystalline Diamond. Phys. Rev. Lett. 2012, 109, 033604.

89. Li, L.; Schröder, T.; Chen, E.H.; Walsh, M.; Bayn, I.; Goldstein, J.; Gaathon, O.; Trusheim, M.E.; Lu, M.; Mower, J.; et al. Coherent spin control of a nanocavity-enhanced qubit in diamond. Nat. Commun. 2015, 6,6173

90. Lee, K.; Chen, X.; Eghlidi, H.; Kukura, P.; Lettow, R.; Renn, A.; Sandoghdar, V.; Götzinger, S. A planar dielectric antenna for directional single-photon emission and near-unity collection efficiency. Nat. Photonics 2011, 5, 166-169.

91. Riedel, D.; Rohner, D.; Ganzhorn, M.; Kaldewey, T.; Appel, P.; Neu, E.; Warburton, R. J.; Maletinsky, P. Low-Loss Broadband Antenna for Efficient Photon Collection from a Coherent Spin in Diamond. Phys. Rev. Appl. 2014, 2, 064011.

92. Le Sage, D.; Arai, K.; Glenn, D.; DeVience, S.; Pham, L.; Rahn-Lee, L.; Lukin, M.; Yacoby, A.; Komeili, A.; Walsworth, R. Optical magnetic imaging of living cells. Nature 2013, 496, 486-489.

93. Marseglia, L.; Hadden, J.P.; Stanley-Clarke, A.C.; Harrison, J.P.; Patton, B.; Ho, Y.L.D.; Naydenov, B.; Jelezko, F.; Meijer, J.; Dolan, P.R.; et al. Nanofabricated solid immersion lenses registered to single emitters in diamond. Appl. Phys. Lett. 2011, 98, 133107.

94. Hausmann, B.J.M.; Choy, J.T.; Babinec, T.M.; Shields, B.J.; Bulu, I.; Lukin, M.D.; Loncar, M. Diamond nanophotonics and applications in quantum science and technology. Phys. Status Solidi A 2012, 209, 1619-1630.

95. Beha, K.; Fedder, H.; Wolfer, M.; Becker, M.C.; Siyushev, P.; Jamali, M.; Batalov, A.; Hinz, C.; Hees, J.; Kirste, L.; et al. Diamond nanophotonics. Beilstein J. Nanotechnol. 2012, 3, 895-908.

96. Aharonovich, I.; Neu, E. Diamond Nanophotonics. Adv. Opt. Mater. 2014, 2, 911-928.

97. Schroder, T.; Mouradian, S.L.; Zheng, J.; Trusheim, M.E.; Walsh, M.; Chen, E.H.; Li, L.; Bayn, I.; Englund, D. Quantum nanophotonics in diamond. J. Opt. Soc. Am. B Opt. Phys. 2016, 33, B65-B83.

98. Kleinlein, J.; Borzenko, T.; Münzhuber, F.; Brehm, J.; Kiessling, T.; Molenkamp, L. NV-center diamond cantilevers: Extending the range of available fabrication methods. Microelectron. Eng. 2016, 159, 70-74.

99. Pelliccione, M.; Jenkins, A.; Ovartchaiyapong, P.; Reetz, C.; Emmanouilidou, E.; Ni, N.; Jayich, A.C.B. Scanned probe imaging of nanoscale magnetism at cryogenic temperatures with a single-spin quantum sensor. Nat. Nanotechnol. 2016, 11, 700-705.

100. Nelz, R.; Fuchs, P.; Opaluch, O.; Sonusen, S.; Savenko, N.; Podgursky, V.; Neu, E. Color center fluorescence and spin manipulation in single crystal, pyramidal diamond tips. Appl. Phys. Lett. 2016, 109, 193105. 
101. Tao, Y.; Boss, J.; Moores, B.; Degen, C. Single-crystal diamond nanomechanical resonators with quality factors exceeding one million. Nat. Commun. 2014, 5, 3638.

102. Jung, T.; Kreiner, L.; Pauly, C.; Mücklich, F.; Edmonds, A.M.; Markham, M.; Becher, C. Reproducible fabrication and characterization of diamond membranes for photonic crystal cavities. Phys. Status Solidi A 2016, 213, 3254-3264.

103. Piracha, A.H.; Ganesan, K.; Lau, D.W.M.; Stacey, A.; McGuinness, L.P.; Tomljenovic-Hanic, S.; Prawer, S. Scalable fabrication of high-quality, ultra-thin single crystal diamond membrane windows. Nanoscale 2016, 8, 6860-6865.

104. Riedrich-Möller, J.; Kipfstuhl, L.; Hepp, C.; Neu, E.; Pauly, C.; Mücklich, F.; Baur, A.; Wandt, M.; Wolff, S.; Fischer, M.; et al. One- and two-dimensional photonic crystal microcavities in single crystal diamond. Nat. Nanotechnol. 2012, 7, 69.

105. Babinec, T.; Hausmann, B.; Khan, M.; Zhang, Y.; Maze, J.; Hemmer, P.; Loncar, M. A diamond nanowire single-photon source. Nat. Nanotechnol. 2010, 5, 195-199.

106. Hausmann, B.; Babinec, T.; Choy, J.; Hodges, J.; Hong, S.; Bulu, I.; Yacoby, A.; Lukin, M.; Lončar, M. Single-color centers implanted in diamond nanostructures. New J. Phys. 2011, 13, 045004.

107. Momenzadeh, S.A.; Stöhr, R.J.; de Oliveira, F.F.; Brunner, A.; Denisenko, A.; Yang, S.; Reinhard, F.; Wrachtrup, J. Nanoengineered Diamond Waveguide as a Robust Bright Platform for Nanomagnetometry Using Shallow Nitrogen Vacancy Centers. Nano Lett. 2015, 15, 165-169.

108. Jiang, Q.; Li, W.; Tang, C.; Chang, Y.; Hao, T.; Pan, X.; Ye, H.; Li, J.; Gu, C. Large scale fabrication of nitrogen vacancy-embedded diamond nanostructures for single-photon source applications. Chin. Phys. B 2016, 25, 118105.

109. Lesik, M.; Tetienne, J.P.; Tallaire, A.; Achard, J.; Mille, V.; Gicquel, A.; Roch, J.F.; Jacques, V. Perfect preferential orientation of nitrogen-vacancy defects in a synthetic diamond sample. Appl. Phys. Lett. 2014, 104, 113107.

110. Michl, J.; Teraji, T.; Zaiser, S.; Jakobi, I.; Waldherr, G.; Dolde, F.; Neumann, P.; Doherty, M.W.; Manson, N.B.; Isoya, J.; et al. Perfect alignment and preferential orientation of nitrogen-vacancy centers during chemical vapor deposition diamond growth on (111) surfaces. Appl. Phys. Lett. 2014, 104, 102407.

111. Fukui, T.; Doi, Y.; Miyazaki, T.; Miyamoto, Y.; Kato, H.; Matsumoto, T.; Makino, T.; Yamasaki, S.; Morimoto, R.; Tokuda, N.; et al. Perfect selective alignment of nitrogen-vacancy centers in diamond. Appl. Phys Express 2014, 7, 055201.

112. Tallaire, A.; Achard, J.; Boussadi, A.; Brinza, O.; Gicquel, A.; Kupriyanov, I.; Palyanov, Y.; Sakr, G.; Barjon, J. High quality thick CVD diamond films homoepitaxially grown on (111)-oriented substrates. Diam. Relat. Mater. 2014, 41, 34-40.

113. Lesik, M.; Plays, T.; Tallaire, A.; Achard, J.; Brinza, O.; William, L.; Chipaux, M.; Toraille, L.; Debuisschert, T.; Gicquel, A.; et al. Preferential orientation of NV defects in CVD diamond films grown on (113)-oriented substrates. Diam. Relat. Mater. 2015, 56, 47-53.

114. Aharonovich, I.; Lee, J.C.; Magyar, A.P.; Bracher, D.O.; Hu, E.L. Bottom-up engineering of diamond micro- and nano-structures. Laser Photonics Rev. 2013, 7, L61-L65.

115. Zhang, X.; Hu, E.L. Templated growth of diamond optical resonators via plasma-enhanced chemical vapor deposition. Appl. Phys. Lett. 2016, 109, 081101.

116. de Oliveira, F.F.; Momenzadeh, S.A.; Wang, Y.; Konuma, M.; Markham, M.; Edmonds, A.M.; Denisenko, A.; Wrachtrup, J. Effect of low-damage inductively coupled plasma on shallow nitrogen-vacancy centers in diamond. Appl. Phys. Lett. 2015, 107, 073107.

117. Lesik, M.; Spinicelli, P.; Pezzagna, S.; Happel, P.; Jacques, V.; Salord, O.; Rasser, B.; Delobbe, A.; Sudraud, P.; Tallaire, A.; et al. Maskless and targeted creation of arrays of colour centres in diamond using focused ion beam technology. Phys. Status Solidi A 2013, 210, 2055-2059.

118. Meijer, J.; Pezzagna, S.; Vogel, T.; Burchard, B.; Bukow, H.; Rangelow, I.; Sarov, Y.; Wiggers, H.; Plümel, I.; Jelezko, F.; et al. Towards the implanting of ions and positioning of nanoparticles with $\mathrm{nm}$ spatial resolution. Appl. Phys. A Mater. Sci. Process. 2008, 91, 567-571.

119. Jacob, G.; Groot-Berning, K.; Wolf, S.; Ulm, S.; Couturier, L.; Dawkins, S.T.; Poschinger, U.G.; Schmidt-Kaler, F.; Singer, K. Transmission microscopy with nanometer resolution using a deterministic single ion source. Phys. Rev. Lett. 2016, 117, 043001. 
120. Bourgeois, E.; Jarmola, A.; Siyushev, P.; Gulka, M.; Hruby, J.; Jelezko, F.; Budker, D.; Nesladek, M. Photoelectric detection of electron spin resonance of nitrogen-vacancy centres in diamond. Nat. Commun. 2015, 6, 8577.

121. Hrubesch, F.M.; Braunbeck, G.; Stutzmann, M.; Reinhard, F.; Brandt, M.S. Efficient Electrical Spin Readout of $\mathrm{NV}^{-}$Centers in Diamond. Phys. Rev. Lett. 2017, 118, 037601.

122. Sekatskii, S.; Dukenbayev, K.; Mensi, M.; Mikhaylov, A.; Rostova, E.; Smirnov, A.; Suriyamurthy, N.; Dietler, G. Single molecule fluorescence resonance energy transfer scanning near-field optical microscopy: Potentials and challenges. Faraday Discuss. 2015, 184, 51-69.

123. Drezet, A.; Sonnefraud, Y.; Cuche, A.; Mollet, O.; Berthel, M.; Huant, S. Near-field microscopy with a scanning nitrogen-vacancy color center in a diamond nanocrystal: A brief review. Micron 2015, 70, 55-63.

124. Cuche, A.; Drezet, A.; Sonnefraud, Y.; Faklaris, O.; Treussart, F.; Roch, J.F.; Huant, S. Near-field optical microscopy with a nanodiamond-based single-photon tip. Opt. Express 2009, 17, 19969-19980.

125. Dexter, D.L. A Theory of Sensitized Luminescence in Solids. J. Chem. Phys. 1953, 21, 836-850.

126. Mohan, N.; Tzeng, Y.; Yang, L.; Chen, Y.; Hui, Y.; Fang, C.; Chang, H. Sub-20-nm Fluorescent Nanodiamonds as Photostable Biolabels and Fluorescence Resonance Energy Transfer Donors. Adv. Mater. 2010, 22, 843-847.

127. Rittweger, E.; Han, K.Y.; Irvine, S.E.; Eggeling, C.; Hell, S.W. STED microscopy reveals crystal colour centres with nanometric resolution. Nat. Photonics 2009, 3, 144-147.

128. Han, K.Y.; Kim, S.K.; Eggeling, C.; Hell, S.W. Metastable Dark States Enable Ground State Depletion Microscopy of Nitrogen Vacancy Centers in Diamond with Diffraction-Unlimited Resolution. Nano Lett. 2010, 10, 3199-3203.

129. Pfender, M.; Aslam, N.; Waldherr, G.; Neumann, P.; Wrachtrup, J. Single-spin stochastic optical reconstruction microscopy. Proc. Natl. Acad. Sci. USA 2014, 111, 14669-14674.

130. Monticone, D.G.; Katamadze, K.; Traina, P.; Moreva, E.; Forneris, J.; Ruo-Berchera, I.; Olivero, P.; Degiovanni, I.; Brida, G.; Genovese, M. Beating the Abbe diffraction limit in confocal microscopy via nonclassical photon statistics. Phys. Rev. Lett. 2014, 113, 143602.

131. Schaefer-Nolte, E.; Reinhard, F.; Ternes, M.; Wrachtrup, J.; Kern, K. A diamond-based scanning probe spin sensor operating at low temperature in ultra-high vacuum. Rev. Sci. Instrum. 2014, 85, 013701.

132. Thiel, L.; Rohner, D.; Ganzhorn, M.; Appel, P.; Neu, E.; Müller, B.; Kleiner, R.; Koelle, D.; Maletinsky, P. Quantitative nanoscale vortex imaging using a cryogenic quantum magnetometer. Nat. Nanotechnol. 2016, 11, 677-681.

133. Kosub, T.; Kopte, M.; Hühne, R.; Appel, P.; Shields, B.; Maletinsky, P.; Hübner, R.; Liedke, M.O.; Fassbender, J.; Schmidt, O.G.; et al. Purely antiferromagnetic magnetoelectric random access memory. Nat. Commun. 2017, 8, 13985.

134. Wrachtrup, J.; Finkler, A. Single spin magnetic resonance. J. Magn. Reson. 2016, 269, 225-236.

135. Mamin, H.J.; Kim, M.; Sherwood, M.H.; Rettner, C.T.; Ohno, K.; Awschalom, D.D.; Rugar, D. Nanoscale Nuclear Magnetic Resonance with a Nitrogen-Vacancy Spin Sensor. Science 2013, 339, 557-560.

136. Staudacher, T.; Shi, F.; Pezzagna, S.; Meijer, J.; Du, J.; Meriles, C.A.; Reinhard, F.; Wrachtrup, J. Nuclear Magnetic Resonance Spectroscopy on a (5 nm) ${ }^{3}$ Sample Volume. Science 2013, 339, 561-563.

137. Lovchinsky, I.; Sanchez-Yamagishi, J.; Urbach, E.; Choi, S.; Fang, S.; Andersen, T.; Watanabe, K.; Taniguchi, T.; Bylinskii, A.; Kaxiras, E.; et al. Magnetic resonance spectroscopy of an atomically thin material using a single-spin qubit. Science 2017, 355, 503-507.

138. Das, T.P.; Hahn, E.L. Nuclear Quadrupole Resonance Spectroscopy; Academic Press Publishers: New York, NY, USA, 1958.

139. Rugar, D.; Mamin, H.J.; Sherwood, M.H.; Kim, M.; Rettner, C.T.; Ohno, K.; Awschalom, D.D. Proton magnetic resonance imaging using a nitrogen-vacancy spin sensor. Nat. Nanotechnol. 2015, 10, 120-124.

140. Häberle, T.; Schmid-Lorch, D.; Reinhard, F.; Wrachtrup, J. Nanoscale nuclear magnetic imaging with chemical contrast. Nat. Nanotechnol. 2015, 10, 125-128.

(C) 2017 by the authors. Licensee MDPI, Basel, Switzerland. This article is an open access article distributed under the terms and conditions of the Creative Commons Attribution (CC BY) license (http:/ / creativecommons.org/licenses/by/4.0/). 